\title{
Langfristig ohne Ekzemschub
}

m Fokus der Grundlagenforschung zum atopischen Ekzem stehen derzeit genetische Ursachen: Über 40 Gene sind beschrieben, bei denen Polymorphismen oder Mutationen für immunologische Defekte, Barrierestörungen und die Überexpression von Entzündungsmediatoren verantwortlich sein könnten. Ihre Relevanz als Zielstrukturen für künftige Therapieregime muss aber erst evaluiert werden.

Indessen habe sich bei der Behandlung von mittelschwerer bis schwerer atopischer Dermatitis die proaktive Therapie in der Praxis und in den Leitlininen etabliert, berichtete Prof. Dr. Thomas Werfel, Hannover, im Rahmen des Allergo-Update-Seminars im Februar dieses Jahres in Wiesbaden. Grundlage der Behandlung ist unabhängig von der Ausprägung der Erkrankung eine konsequente Basistherapie mit Emollenzien. Darüber hinaus wird in einem europäischen Positionspapier die proak- tive Therapie mit dem topischen Calcineurin-Inhibitor Tacrolimus empfohlen. Dabei wird Protopic ${ }^{\circledR}$-Salbe längerfristig zweimal pro Woche auf zuvor befallene Hautareale aufgetragen. Dieser Behandlungsansatz ermöglicht es, in den subklinischen Entzündungsprozess zwischen den Ekzemschüben einzugreifen. Die Zahl neuer Schübe wird signifikant reduziert, die läsionsfreien Intervalle werden verlängert.

Werfel machte die Wirksamkeit anhand von klinischen Studien mit 183 Erwachsenen und 166 Kindern deutlich (J Dermatol Treat 2010; 21: 34-44). Darin wurde die Zeit bis zum ersten Ekzemschub unter der reaktiven Therapie im Vergleich zur proaktiven Therapie mit Tacrolimus bestimmt. Bei Erwachsenen betrug die Zeit bei 0,1\%iger Wirkstoffkonzentration der Salbe hier 15 Tage unter reaktiver Therapie und 142 Tage unter proaktiver Therapie. Bei Kindern waren es bei 0,03\%iger Wirk- stoffkonzentration 36 Tage gegenüber 217 Tagen. Unter der proaktiven Therapie blieben sogar 48,3\% der Erwachsenen über den gesamten Beobachtungszeitraum von einem Jahr ohne Schub - im Vergleich zu 19,4\% bei der reaktiven Therapie. Bei Kindern waren die Verhältnisse ähnlich mit 40,2\% gegenüber $21,3 \%$.

Dabei wurde die Therapie gut vertragen. Werfel betonte, dass es zehn Jahre nach der Zulassung von Tacrolimus und dem funktionsverwandten Wirkstoff Pimecrolimus keine Evidenz für ein erhöhtes Risiko maligner Erkrankungen unter der Therapie gebe. Derartige Hinweise gingen weder aus den prospektiven Beobachtungsstudien noch aus Analysen der Patientendatenbanken hervor. Im Gegenteil sei die Anzahl der unter einer topischen Therapie mit Tacrolimus aufgetretenen Lymphome sogar geringer als es in der Allgemeinbevölkerung zu erwarten wäre. red

Nach Informationen von Astellas Pharma, München

\section{Molkenhydrolysat: Atopieschutz für's Baby}

edes dritte Neugeborene in Deutschland habe ein familiäres Allergierisiko, berichtete Dr. Andrea von Berg, Wesel. Ausgelöst würden überschießende Immunreaktionen wie die atopische Dermatitis jedoch meist erst durch Nahrungsmittelallergene, vor allem das Eiweiß der Kuhmilch. Die Fachärztin für Kinder- und Jugendmedizin empfahl deshalb, allergiegefährdete Babys, die nicht ausreichend gestillt werden können, in den ersten vier Lebensmonaten mit wissenschaftlich geprüfter hypoallergener Anfangsnahrung zu versorgen. Das Protein dieser Babymilch wird in kleinere Peptide aufgespalten, so dass es seine antigenen Eigenschaften verliert. Dass hypoallergene Nahrung Säuglinge und Kleinkinder langfristig vor atopischer Dermatitis schützen kann, zeigte u. a. die vom Bundesforschungsministerium geförderte, weltweit größte Studie zur alimentären Allergieprävention: German
Infant Nutritional Intervention (GINI). In dieser prospektiven doppelblinden Interventionsstudie wurden drei verschiedene Eiweißhydrolysate sowie her-

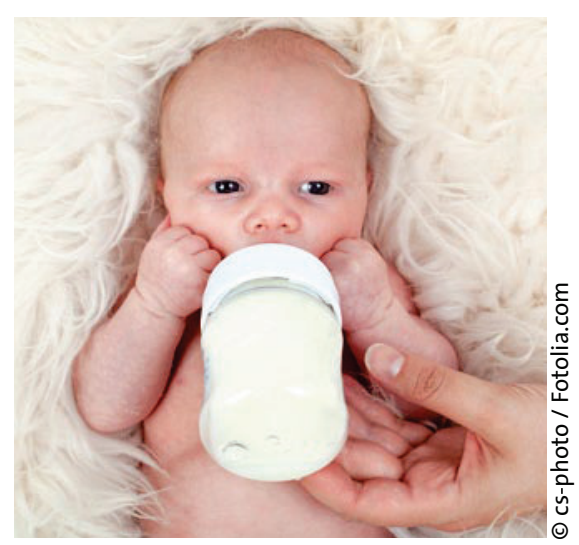

Die Haut von allergiegefährdeten Säuglingen, die nicht gestillt werden können, braucht besonderen Schutz: Hypoallergene Anfangsnahrung kann das Risiko einer atopischen Dermatitis halbieren. kömmliche Säuglingsmilch unter die Lupe genommen. Ergebnis: Alle drei Nahrungen konnten das familiär bedingte zweifach erhöhte Risiko, an einer atopischen Dermatitis zu erkranken, in unterschiedlichem Ausmaß kompensieren. Dagegen wurde es durch die reguläre Kuhmilchformula weiter erhöht. In der GINI-Studie und zwei kürzlich publizierten Metaanalysen schnitt das teilweise aufgespaltene Molkenhydrolysat der Firma Nestlé (Beba ${ }^{\circledR}$ H.A.) besonders günstig $a b$ : Im Vergleich zur Standard-Babynahrung reduzierte es das Risiko einer atopischen Dermatitis signifikant vom Säuglings- $(-52 \%)$ bis ins Schulalter (-36\%). Auch wenn hypoallergene Nahrung zunächst teurer ist: Langfristig können Kosten gesenkt und den betroffenen Familien viel Leid erspart werden, resümierte von Berg.

Dr. Martina-Jasmin Utzt

Expertengespräch „Allergieprävention braucht wissenschaftliche Evidenz". Biessenhofen, 31. März 2011. Veranstalter: Nestlé, Frankfurt/Main 